\title{
UNIVERSAL EXTRA DIMENSIONS AND SUPERSYMMETRY AT THE HL/HE- LARGE HADRON COLLIDER
}

\author{
*Durmuş KARABACAK \\ Department of Energy Systems Engineering, Faculty of Technology, Muğla Sitkı Koçman University, Turkey \\ durmuskarabacak@gmail.com \\ (iD) https://orcid.org/0000-0002-7712-4357
}

Received: 30.01.2018, Accepted: 22.05.2018

*Corresponding author

Research Article

DOI:10.22531/muglajsci.419831

\begin{abstract}
Universal Extra Dimensional (UED) and Supersymmetric (SUSY) models show very similar signals at hadron colliders. In the minimal UED (mUED) where the mass spectrum is compressed, the production of level-1 KK quarks and/or gluons produce multi-jet and/or multi-lepton + missing transverse momenta final state as in the case of SUSY models with R-parity conservation and compressed mass spectrum. Moreover, this similarity can possibly be carried over to non-degenerate mass spectra in next-to-minimal UED (nmUED) model that is the extension of the mUED model with fermion bulk mass term $\mu$ and brane localized kinetic terms $r$, hence, completing the faking in both models. In this regard, we simulated the production of colored level-1 KK particles (quarks and gluons) and colored sparticles (squarks and gulinos) with their decays resulting in multi-jet + missing transverse momenta signals in both models at $14 \mathrm{TeV}$ LHC. Then we generated normalized m_effdistributions originating from multi-jet final state in both models and compared. We found that the similarity of both models is indeed the case even with non-compressed mass spectra though there are some fluctuations, in the distributions for some signal regions, resulting from low statistics.

Keywords: The Large Hadron Collider, Universal Extra Dimensions, Supersymmetry, Phenomenology

\section{HL/HE BÜYÜK HADRON ÇARPIŞTIRICISIN'DA EVRENSEL ÇOK BOYUTLAR VE SÜPERSIMETRI}

Öz

Evrensel Çok Boyutlu (UED) ve Süpersimetrik (SUSY) kuramlar hadron çarpıştırıcılarında benzer sinyaller gösterirler. Parçacık spektrumunun sıkışık olduğu kısmi UED'de (mUED), R-paritesi'nin korunduğu ve sıkışık kütle durumuna spektrumuna sahip SUSY modellerinde olduğu gibi, 1. seviye Kaluza-Klein (KK) gluon ve kuarklar son durumda çoklu jet ve/veya çoklu lepton + kayıp transvers momentum üretirler. Dahası bu benzerlik durumu, fermiyon yığın kütle terimi $\mu$ ve zara sınırlı kinetik terimleri r ile mUED'nin bir uzantısı olan nmUED modeli vasıtasılya, sı kışık olmayan kütle spektrumuna da taşınması imkânı olup, bu sayede benzeşme her durumda bütünlük göstermektedir. Bu bağlamda, 1. seviye renkli KK parçacıklarını (kuark ve gulion) ve sparçacıkar (skuark ve gulino) 14 TeV LHC'de üretimini ve bozunumunu çoklu jet + kayıp transvers momentum sinyalini üretmek suretiyle simüle ettik. Daha sonra, her iki modelden de gelen çoklu-jet son durumundan normalize edilmiş m_effdağılımlarını üretip karşılaştırdık. Her iki modelin benzeştiğini ve benzeşmenin, kimi sinyal bölgelerinde düşük istatistikten dolayı dalgalanmalar olmasına rağmen, ayrık kütle spektrumunda dahi gerçekleştiğini bulduk.

Anahtar Kelimeler: Büyük Hadron Çarpıştırıcısı, Evrensel Çok Boyutlar, Süpersimetri, Fenomenoloji

Cite

Karabacak, D., (2018). “Universal extra dimensions and supersymmetry at the HL/HE-large hadron collider", Mugla Journal of Science and Technology, 4(1), 53-63.

\section{Introduction}

The Large Hadron Collider (LHC) experiments at CERN are continuing searches which may shed a light on TeV- scale new physics. In this regard a lot of attention is put on Supersymmetric (SUSY) and extra dimensional models both theoretically and experimentally. SUSY models are famous for various reasons. It addresses hierarchy problem regarding the quadratic divergencies in the Higgs mass, offers a cold dark matter candidate if the R-parity is conserved, and predicts $\mathrm{TeV}$ scale new particles which could be observed at collider experiments [1]. Although the collider results for SUSY are null so far $[2,3]$, expectations for upgraded LHC are high $[4,5]$.

Extra dimensional models are also of interest for a variety of reasons. Arkani-Dimopoulos-Dvali [6] and Randall-Sundrum [7] models offer a framework in which the long-standing hierarchy problem of SM can be addressed. In this type of models, one assumes $4+D$ dimensional space-time and allows only the gravity to access the full bulk. In these models the Standard Model (SM) particles reside on $3+1$ dimensional 3brane. 
In other realizations of extra dimensional models, one assumes that all the SM particles can access the full spacetime. One of the famous examples of these types of models is the Universal Extra Dimensions (UED) [8-11]. In its minimal realization, the minimal Universal Extra Dimensions (mUED) [8-12], one assumes the existence of a flat extra dimension $(y)$ which is accessible to all SM particles. The extra dimension is compactified on a circle $S_{1}$ of $\sim \mathrm{TeV}^{-1}$ radius $R$ along with addition of $Z_{2}$ symmetry which identified $y \rightarrow-y$ on $S_{1}$ to get the chiral structure of the SM. The SM gauge group $\left(S U(3)_{C} \times\right.$ $\left.S U(2)_{L} \times U(1)_{Y}\right)$ is preserved. Translational invariance broken by $Z_{2}$ along the extra dimension creates two fix points at $y=$ $0, \pi R$. Each field present in higher dimensional Lagrangian is then decomposed into infinite number of modes identified by Kaluza-Klein (KK) number, $n . n=0$ is identified with the corresponding SM particle and $n>0$ corresponds to new massive excitations of SM particles. In mUED, at the tree level, the KK-number is conserved. However, the $Z_{2}$ symmetry breaks KK-number down to KK-parity (defined as $\equiv(-1)^{n}$ ). At tree level, the mass of nth-level Kk particle is $\sim n R^{-1}$ and, hence the mass spectrum is quite degenerate. However, as UED models are higher dimensional and therefore non-renormalizable, it is assumed that the model is valid up to a cut-off scale $\Lambda$ at which new dynamics take place. The choice of $\Lambda=5 R^{-1}$ is assumed throughout our analysis. Radiative corrections [13] can lift this degeneracy to some degree to allow various decay channels. The KK-parity in the model forbids the decay of lowest lying KK particle in the spectrum and hence gives a viable cold dark matter candidate [14-16]. The level-1 KK excitation of SM particles is quite similar to that of R-parity SUSY models with a compress mass spectrum. Therefore, UED models sometimes are referred as bosonic SUSY [12].

A possible extension of UED Lagrangian is the addition of bulk mass terms for 5D fermions. These terms respect the 5D Lorentz invariance and gauge symmetry of the SM. This extension of mUED is called as split-UED (sUED) and its phenomenological consequences are discussed in literature [17-24]. Introduction of these terms modifies KK fermion profile, KK particle masses at the tree-level and their couplings with SM gauge bosons. The KK gauge bosons masses, however, are not affected and stays the same as in the mUED. Another possible extension is so-called non-minimal UED (nUED) [2529]. This extension assumes that brane localized kinetic terms (BLKT) at orbifold fixed points $y=0, \pi R$ are non-vanishing. BLK terms respect 4D Lorentz invariance and the SM gauge group. Another extension, which assumes the presence of both terms (fermion bulk mass and BLKT) simultaneously, is called as next-to-minimal UED (nmUED), has also been investigated in the literature [30]. With this setup nmUED has four free parameters $R$ size of the extra dimension, $\Lambda$ the cut-off scale of the model, $\mu$ the fermion bulk mass and $r$ the brane localized kinetic term coefficient. Consequence of the extra terms in nmUED is to modify mass spectra of KK- particles and their couplings significantly compared to the mUED. Hence, the faking of compressed SUSY model signal to mUED can also be a problem in the case of non- compressed SUSY mass spectra. In this work, we investigate the faking in UED and SUSY models by comparing the production and decay of colored particles (in the case of MSSM squarks and/or gluinos, and in nmUED level1 quarks and/or gulinos) at the $14 \mathrm{TeV}$ LHC. In doing so, we assume a more general, non-compressed mass spectra for both models and analyze the signal in multi-jet + large missing transverse momenta final state. We show the similarity between two models by producing normalized $m_{\text {eff }}$ distributions which is the main kinematic variable in SUSY model searches at the LHC, in three benchmark points in mass spectra.

The paper is organized as following. We give a brief description of the model in Section 2. Then, we compare the signals coming from nmUED and MSSM models and present our result in Section 3. In Section 4 we discuss and conclude. Appendix contains the mass spectra of particles in our benchmark points and normalized $m_{e f f}$ distributions corresponding to these points.

\section{Material and Method}

\subsection{The Model}

The model under consideration is given in details in [30]. We only present key features of the model and summarize it in here. The nmUED model Lagrangian for gauge bosons and fermions is consisted of two parts. The first part is originating from the bulk interactions:

$$
=\int_{-L}^{L} d y\left(\sum_{\mathcal{A}}^{G, W, B} \frac{-1}{4} \mathcal{A}^{M N} \mathcal{A}_{M N}+\sum_{\Psi}^{\mathcal{L}_{\text {bulk }}} i \sum_{\left.-M_{\Psi} \bar{\Psi} \Psi\right),}^{Q, U, D, L, E}, \overleftrightarrow{\Phi}_{M} \Gamma^{M} \Psi\right.
$$

where the extra dimension is compactified on a half circle $S_{1} / Z_{2}$ with a length of $2 L$ (or equivalently $\pi R$ ). $\mathcal{A}, \Psi$ and $M_{\Psi}=\mu \theta(y)$ are 5D gauge boson strength tensors, fermions and fermion bulk mass terms with $\theta(y)$ being the step function. 5D gamma matrices are $\Gamma^{M}=\left(i \gamma^{\mu}, i \gamma^{5}\right)$ as usual and $\bar{\Psi} \overleftrightarrow{D}_{M} \Psi=1$ / $2\left\{\bar{\Psi} D_{M} \Psi-\left(D_{M} \bar{\Psi}\right) \Psi\right\}$.

The second part comes from the interactions present at the orbifold fixed points at $y=-L, L$ with $L=\pi R / 2$ :

$$
\begin{aligned}
& \mathcal{L}_{\text {bdry }}=\int_{-L}^{L} d y\left(\sum_{\mathcal{A}}^{G, W, B}-\frac{r_{\mathcal{A}}}{4} \mathcal{A}_{\mu \nu} \mathcal{A}^{\mu \nu}\right. \\
&+\sum_{\Psi_{L}}^{Q, L} i r_{\Psi} \bar{\Psi}_{L} D_{\mu} \gamma^{\mu} \Psi_{L} \\
&\left.+\sum_{\Psi_{R}}^{U, D, E} i r_{\Psi} \bar{\Psi}_{R} D_{\mu} \gamma^{\mu} \Psi_{R}\right) \\
& \times[\delta(y-L)+\delta(y+L)],
\end{aligned}
$$

where $r_{i}$ with $i=\mathcal{A}, \Psi$ is the BLK terms for 4D gauge bosons and fermions present at the orbifold fixed points. Once the Fourier expansion of 5D fields is performed and integrated over $y$ to obtain the interactions in $4 \mathrm{D}$ one can see that there is an infinite tower of massive particles with masses increasing with each mode. In comparison to mUED where the mass of KKgauge boson at the level-n is given by $\sim n R^{-1}$, it is the wave number, $k_{n}$, obtained as solution of the following transcendental equations:

$$
\begin{aligned}
& \cot \left(k_{(2 j-1)} L\right)-r_{\mathcal{A}} k_{(2 j-1)}=0, \text { for } n=\text { odd } \\
& \tan \left(k_{(2 j)} L\right)+r_{\mathcal{A}} k_{(2 j)}=0, \text { for } n=\text { even. }
\end{aligned}
$$

In the case of KK-fermions the wave numbers $k_{n}$, are determined from following equations:

$$
\begin{aligned}
& k_{n} \mathcal{C}_{n}(L)-\left(r_{\Psi} m_{f_{n}}^{2}+\mu\right) \mathcal{S}_{n}(L)=0, \text { for odd KK modes, } \\
& r_{\Psi} k_{n} \mathcal{C}_{n}(L)+\left(1+r_{\Psi} \mu\right) \mathcal{S}_{n}(L)=0 \text {, for even KK modes }
\end{aligned}
$$

where $\mathcal{C}_{n}(x)=\cos \left(k_{n} x\right)$ and $\mathcal{S}_{n}(x)=\sin \left(k_{n} x\right)$. The mass of level- $n$ KK fermion $m_{f_{n}}$ is then given by $m_{f_{n}}=\sqrt{k_{n}^{2}+\mu^{2}}$ with extra contribution from fermion bulk mass term $\mu$. There are 
various constraints on the parameters of nmUED. For instance, decomposition of wave functions of KK-gauge bosons dictates that $r_{\mathcal{A}} / L>-1$. Similarly, for KK-fermions $r_{\Psi} / L>\left(e^{-\mu L}-\right.$ 1) $/ 2 \mu L$ should be satisfied to avoid ghosts and/or tachyons in the model [30],[40]. Experimentally, there are bounds from low-energy observables and collider searches. For example, KK-number conserving but KK-number violating interactions in the form of $Z_{\mu}^{(2)} \bar{\psi}^{(0)} \psi^{(0)}$ contributes to the four-Fermi interactions even at tree level. However, although the coupling of this interaction is $g_{200}$ is non-zero, in the vicinity of $\mu=0$ the interaction strength will be very small (around $\sim 1 \%$ of SM coupling). With a modest value of $R^{-1} \simeq 900 \mathrm{GeV}$ for $0>\mu L>$ $-0.03, g_{200}$ and $Z_{\mu}^{(2)}$ will be small and heavy enough to evade this constrain. From the collider physics side, the search on $Z^{\prime}$ like particles decaying into leptons (electron and muons) has been performed by the CMS collaboration. The bound of this and similar searches can be well applied to level-2 gauge bosons in UED models as a lower bound on their masses. On the other hand, as above-mentioned any search performed will not be sensitive for $\mu$ in the vicinity of 0 . Therefore, in this work we have chosen a global value of $\mu=-0.02 L$ throughout our analysis for KK-leptons and quarks. The details of the bounds can be reached at Ref. [30] and [40].

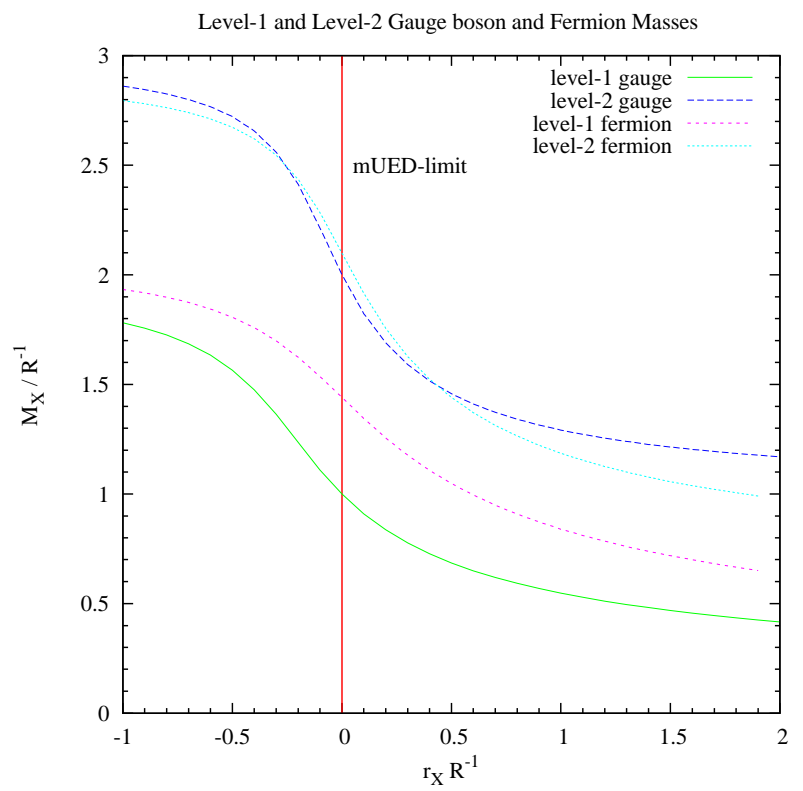

Figure 1 Normalized masses of 1 st and 2nd level gauge bosons and fermions as a function of $r / R$ for three different values of $R^{-1}$. For KK-fermions masses, we consider $\mu=-1 / L$. Solid line extending vertically corresponds to mUED limit. We set $\Lambda=5 R^{-1}$ in the plot.

At this point we'd like to point out that the mass spectrum of nmUED model can be more hierarchical than what one would obtain in mUED, and this is still the case even with inclusion of radiative corrections. The reason is that the extra parameters, in particular, BLK terms for KK-gauge bosons and fermions (mainly, quarks in this work) can be different which results in the separation of their masses. To give a better sense of this phenomena, we plotted normalized masses of KK particles (level-1 and 2 gauge bosons and fermions) in Fig. 1. In Fig. 1 vertical solid line at $r_{X} R^{-1}=0$ corresponds to mUED limit with $\mu=0$ and $r=0$.

\subsection{Non-degenerate Mass Spectrum}

SUSY and UED models both predict heavy TeV scale colored particles having high production rates at the LHC. In the case of
Minimal Supersymmetric Standard Model (MSSM) where the Rparity is conserved, the production of squarks $\tilde{q}$ and gluinos $\tilde{g}$ at the LHC can result in multi-jet and/or multi-lepton + missing transverse momenta final state. This happens through the decay of $\tilde{q}, \tilde{g}$ into lighter sparticles and SM particles. The decay cascade stops at the Lightest SUSY particles (LSP). As LSP is weakly interacting and stable, it is recorded as a missing energy signature in the detector. Therefore, SUSY models with R-parity conservation predict multi-jet and/or multi-lepton + missing transverse energy final state at the LHC. Similar scenario is also seen in UED models where the strong production of level-1 KK quarks $\left(q^{(1)}\right)$ and gluons $\left(g^{(1)}\right)$ results in cascade decay of these particles into lighter level-1 particles and a SM particle. As in MSSM the cascade decay stops at lightest KK particle (LKP). Just like LSP, LKP is also weakly interacting and stable, escapes from detection and is recorded as missing transverse energy in the events. The ATLAS Collaboration [31] has searched for SUSY particles in the multi-jet + MET final state at $\sqrt{s}=13 \mathrm{TeV}$. The ATLAS collecting $36.1 \mathrm{fb}^{-1}$ of data, in the absence of signal above the SM background, excluded gluinos with a mass below 2.03 TeVat the $95 \%$ C.L. in a simplified model incorporating only gluinos and massless neutralino. The analysis also excluded squarks with mass below $1.58 \mathrm{TeV}$ assuming strong production of first two generation of squarks with their decay into massless neutralino LSP. The ATLAS search consists of two complementary ' $M$ eff -based search' and 'Recursive Jigsaw Reconstruction' techniques, where the latter is useful for a search with compressed SUSY mass spectrum.

\section{Results}

In this work, in order to show that SUSY and UED models give a similar signature, even in the case of non-compressed spectra, we used simulation studies performed by the ATLAS [32] for high luminosity/high energy HL/HE-LHC. In that study, simplified models of gluinos and squarks pair-production is considered. Gluinos are assumed to decay into two SM quarks and neutralino $\tilde{\chi}_{1}^{0}$ with $100 \%$ branching ratio (BR), and similarly, squarks decay into SM quarks and neutralino $\tilde{\chi}_{1}^{0}$ with $100 \%$ BR. In both cases, the signal is characterized by multi-jet + large MET and no leptons. Before going into our results, we would like to discuss the details of the ATLAS simulation study. The study was performed at $\sqrt{s}=14 \mathrm{TeV}$ center-of-mass energy with 300 and $3000 \mathrm{fb}^{-1}$ integrated luminosities on $2-$ 6 jets + MET final state. Jets are reconstructed with anti- $k_{T}$ algorithm with a radius parameter $\Delta R=0.4$. Jets are selected with $p_{T}>20 \mathrm{GeV}$ and within a rapidity coverage of $|\eta|<4.5$. Electron (muon) candidates are required to satisfy $p_{T}>$ 20 (10) GeV and $|\eta|<2.47(2.4)$. Following these steps, overlap between candidate jets with $|\eta|<2.8$ and leptons (electron or muon) are resolved as follows. Any jet candidate lying within a distance $\Delta R=\sqrt{\Delta \eta^{2}+\Delta \phi^{2}}<0.2$ of an electron is discarded. Then any lepton candidate remaining within a distance $\Delta R=$ 0.4 of any surviving jet candidate is discarded. The measurement of two-dimensional MET vector $\boldsymbol{E}_{T}^{\text {miss. }}$ (and its magnitude $E_{T}^{\text {miss. }}$ ) is reconstructed by using all remaining jets and leptons and all calorimeter clusters not associated with such objects. Following the calculation of MET, all jet candidates with $|\eta|>2.8$ are removed from the list. After the object reconstruction, events are discarded if they contain any lepton (electron or muon) with $p_{T}>10 \mathrm{GeV}$. In order to analyze the signal, multiple signal regions (SR) with increasing jet multiplicity are defined. The optimization on the SRs is achieved with the requirement on the effective mass $m_{\text {eff }}=$ $M E T+\sum\left|p_{T}^{j e t}\right|, M E T / m_{\text {eff }}$ and $M E T / \sqrt{H_{T}}$ kinematic variables where $H_{T}$ is defined as the scalar sum of transverse momenta 
of all jets in the event. We present the SRs and kinematic cuts used by the ATLAS in Table 1 in Appendix. In order to show the similarity between two models, we choose three benchmark points (BP) presented in Table 2. The BPs are generated by SUSPECT [33] SUSY particle spectrum generator for sparticles and passed to PYTHIA [34] to simulate $\tilde{q} \tilde{q}, \tilde{q} \tilde{g}$ and $\tilde{g} \tilde{g}$ production at the LHC. For nmUED we have used CalcHEP [35] with its UED implementation [36] to generate mass spectra and passed to PYTHIA [34] through SLHA-BSM [37] convention to simulate $q^{(1)} q^{(1)}, q^{(1)} g^{(1)}$ and $g^{(1)} g^{(1)}$ production as well as their decays, initial/final state radiation and hadronization etc. In presenting the results we plotted normalized $m_{\text {eff }}$ distributions coming from the signals of SUSY and nmUED models with mass spectra given in Table 2. Our results are given in Figure 2-6 for BP 1 and 3 in $2-6$ jet + MET signal topologies ${ }^{1}$. Despite of the fact that there is a fluctuation in $6 \mathrm{~J}-\mathrm{T}$ SR due to lack of statistics, on can clearly state from the figures that nmUED and SUSY models with non-degenerate mass spectra generates a very similar signature in all SRs and it is not possible to distinguish one from another by using current search strategies performed by ATLAS $^{2}$ and CMS Collaborations. This conclusion may not be a surprise as it has been shown that both models (actually mUED and MSSM) with degenerate mass spectra show strikingly similar signatures [12] at hadron colliders. The results presented here are confirmation of extension of this phenomenon to nondegenerate mass spectrum which has not been shown quantitatively so far. In spite of the fact that both models show similar signatures in hadron colliders in hadron colliders there are attempts to distinguish one from another [38]. For instance, new particles in both models differ by their spin quantum number. This information can be used to construct a new variable (charge asymmetry) which can be used to differentiate both models. However, due to lack of statistics it is not possible to conclusively differentiate both models at hadron colliders.

\section{Conclusion}

In this work, we have investigated the similarity of the collider phenomenology of level-1 KK quarks $q^{(1)}$ and gluons $g^{(1)}$ in next-to-minimal Universal Extra Dimensions (nmUED) and squarks $\tilde{q}$ and gluinos $\tilde{g}$ in minimal Supersymmetric Standard Model (MSSM) at the LHC. Just like the collider phenomenology similarity between minimal Universal Extra Dimensions (mUED) and MSSM which is observed in the case of compressed (degenerate) mass spectrum, the similarity has been shown in a more general, non-compressed mass spectrum case. In doing this, we compared normalized $m_{\text {eff }}$ distributions which are the main search kinematic in the search of colored SUSY particles at the LHC the ATLAS and CMS Collaboration. The noncompressed spectrum in nmUED is achieved by two additional parameters not present in mUED. These parameters are, bulk mass term $\mu$ for 5D fermions and brane localized kinetic terms (BLKT) at orbifold fixed points can dramatically change the mass spectra of Kaluza-Klein particles in the nmUED and hence completes the faking in a more general setup.

\section{Acknowledgment}

The author thanks to Satya Nandi for initiating the project and to Kirtiman Ghosh for useful discussions and also for the help on PYTHIA. The work of the author was supported in part by

\footnotetext{
${ }^{1}$ Although it is not shown in this paper Benchmark Point-2 also shows a very similar $m_{\text {eff }}$ distribution for nmUED and SUSY models in all Signal Regions.
}

TÜBİTAK, the Scientific and Technical Research Council of Turkey through 2232 program, Project no: 115C058.

\section{References}

[1] S. P. Martin, "A supersymmetry primer", https://arxiv.org/abs/hep-ph/9709356.

[2] The ATLAS Collaboration 2018. Summary plots from the ATLAS Supersymmetry physics group, https://atlas.web.cern.ch/Atlas/GROUPS/PHYSICS/com binedSummaryPlots/SUSY/

[3] The CMS Collaboration 2018. CMS Supersymmetry Physics Results. https://twiki/cern.ch/twiki/bin/view/CMSPublicResult sSUS

[4] The ATLAS Collaboration 2014." Search for Supersymmetry at the high luminosity LHC with the ATLAS experiment". https://cds.cern.ch/record/1735031/files/ATL-COMPHYS-2014-555.pdf

[5] The CMS Collaboration 2015. "Supersymmetry discovery potential in future LHC and HL-LHC running with the CMS detector".

https://cds.cern.ch/record/1981344/files/SUS-14-012pas.pdf

[6] Arkani-Hamed N., Dimopoulos S. and Dvali G. R. "The Hierarchy problem and new dimensions at a millimeter", Physics Letters B, 429, 263-272, 1998.

[7] Randall L. and Sundrum R. "A Large mass hierarchy from a small extra dimension", Physical Review Letters, 83, 3370-3373, 1999.

[8] Appelquist T., Cheng H. C. and Dobrescu B. A., "Bounds on universal extra dimensions”, Physical Review D, 64, 035002, 2001.

[9] Macesanu C., McMullen C. D. and Nandi S., "Collider implications of universal extra dimensions", Physical Review D, 66, 015009, 2002.

[10] Macesanu C., McMullen C. D. and Nandi S., "New signals for universal extra dimensions”, Physics Letters B, 546, 253, 2002.

[11] Macesanu C., McMullen C. D. and Nandi S., "Collider implications of models with extra dimensions", ICHEP 2002, 2002, 764-766.

[12] Cheng H. C., Matchev K. T. and Schmaltz M., "Bosonic supersymmetry? Getting fooled at the LHC", Physical Review D, 66, 056006, 2002.

[13] Cheng H. C., Matchev K. T. and Schmaltz M., "Radiative corrections to Kaluza-Klein masses", Physical Review D, 66, 036005, 2002.

[14] Servant G. and Tait T. M. P., "Is the lightest Kaluza-Klein particle a viable dark matter candidate?", Nuclear Physics B, 650, 391-419.

[15] Cheng H. C., Feng J. L. and Matchev K. T., "Kaluza-Klein dark matter", Physical Review Letters, 89, 211301, 2002.

[16] Kong K. and Matchev K. T., "Precise calculation of the relic density of Kaluza-Klein dark matter in universal extra dimensions", Journal of High Energy Physics, 0601, 038, 2006.

[17] Kong K, Park S. C. and Rizzo T. G., "Collider Phenomenology with Split-UED", Journal of High Energy Physics, 1004, 081, 2010.

2 In Ref. [31] the ATLAS Collaboration used an additional kinematic variable called as aplanarity. We note that although this variable can reduce the SM background in SUSY searches, it nevertheless help distinguish one model from another. 
[18] Kim D. Oh Y. and Park S. C., " $W$ ' at the LHC with $\sqrt{s}=14$ TeV: Split universal extra dimension model", Journal of the Korean Physical Society, 67, 1137-1141, 2015.

[19] Flacke T. and Pasold C., "Constraints on split-UED from Electroweak Precision Tests", Physical Review D, 85, 126007, 2012.

[20] Chen C. R., Nojiri M. M., Park S. C., Shu J. and Takeuchi M., "Dark matter and collider phenomenology of split-UED", Journal of High Energy Physics, 0909, 078, 2009.

[21] Kong K., Park S. C. and Rizzo T. G., "A vector-like fourth generation with a discrete symmetry from Split-UED", Journal of High Energy Physics, 1007, 059, 2010.

[22] Huang, G. Y., Kong K. and Park S. C., "Bounds on the Fermion-Bulk Masses in Models with Universal Extra Dimensions", Journal of High Energy Physics, 1206, 099, 2012.

[23] Park S. C. and Shu J, "Split Universal Extra Dimensions and Dark Matter", Physical Review D, 79, 091702, 2009.

[24] Kong K., Park S. C. and Rizzo T. G., "Collider Phenomenology with Split-UED", Journal of High Energy Physics, 1004, 081, 2010.

[25] Dvali G. R., Gabadadze G., Kolanovic M. and Nitti F. "Power of brane-induced gravity”, Physical Review D, 64, 084004, 2001.

[26] Carena M., Tait T. M. P. and Wagner C. E. M., "Branes and orbifolds are opaque”, Acta Physica Polonica B, 33, 2355.

[27] Aguila del F., Victoria-Perez M. and Santiago J., "Effective description of brane terms in extra dimensions", Journal of High energy Physics, 0610, 056, 2006.

[28] Aguila del F., Victoria-Perez M, and Santiago J., "Bulk fields with general brane kinetic terms", Journal of High Energy Physics, 0302, 051, 2003.

[29] Flacke T., Menon A. and Phalen D. J., "Non-minimal universal extra dimensions", Physical Review D, 79, 056009, 2009.

[30] Flacke T., Kong K. and Park S. C., "Phenomenology of Universal Extra Dimensions with Bulk-Masses and BraneLocalized Terms", Journal of High Energy Physics, 1305, 111, 2013.

[31] The ATLAS Collaboration, "Search for squarks and gluinos in final states with jets and missing transverse momentum using $36 \mathrm{fb}^{-1}$ of $\sqrt{\mathrm{s}}=13 \mathrm{TeV}$ pp collisions data with the ATLAS detector", http://cdsweb.cern.ch/record/2258145.

[32] The ATLAS Collaboration, "Search for Supersymmetry at the high luminosity LHC with the ATLAS experiment", https://atlas.web.cern.ch/Atlas/GROUPS/PHYSICS/PUB NOTES/ATL-PHYS-PUB-2014-010/.

[33] Djouadi A. Kneur J. L. and Moultaka G., "SuSpect: A Fortran code for the supersymmetric and Higgs particle spectrum in the MSSM", Computer Physics Communications, 176, 426, 2007.

[34] Sjostrand T., Mrenna S. and Skands P. Z., "PYTHIA 6.4 Physics and Manual", Journal of High Energy Physics, 0605, 026, 2006.

[35] Pukhov A., "CalcHEP 2.3: MSSM, structure functions, event generations, batches, and generation of matrix elements for other packages", https://arXiv.org/abs/hep$\mathrm{ph} / 0412191$.

[36] Datta A., Kong K. and Matchev K. T., "Minimal Universal Extra Dimensions in CalcHEP/CompHEP", New Journal of Physics, 12, 075017.
[37] Alwall J. et al. "A Les Houches Interface for BSM Generators", https://arXiv.org/abs/0712.3311.

[38] Datta A., Kong K. and Matchev K. T., "Discrimination of supersymmetry and universal extra dimensions at hadron colliders", Physical Review D, 72, 096006, 2005. [Erratum: Phyical Review D, 72, 119901, 2005].

[39] Pumplin J., Stump D. R., Huston J., Lai H. L., Nadolsky P. M. and Tung W. K., "New generation of parton distributions with uncertainties from global QCD analysis", Journal of High Energy Physics, 0207, 012, 2002.

[40] Ghosh K., Karabacak D. and Nandi S., "Constraining bosonic supersymmetry from Higgs results and $8 \mathrm{TeV}$ ATLAS multi-jets plus missing energy data", Journal of High Energy Physics, 09, 076, 2014. 


\section{Appendix A}

In this Appendix we present the cut-table of ATLAS Collaboration [4] in Table 1 and the mass spectrum for nmUED and R-parity conserving SUSY (actually MSSM) model in Table 2.
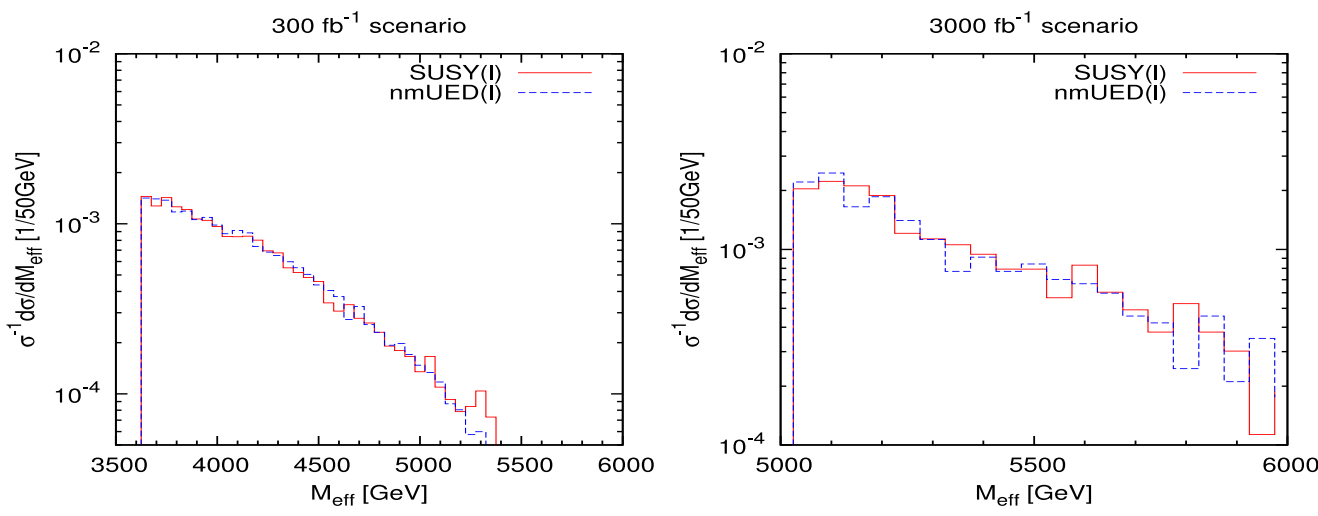

(a)
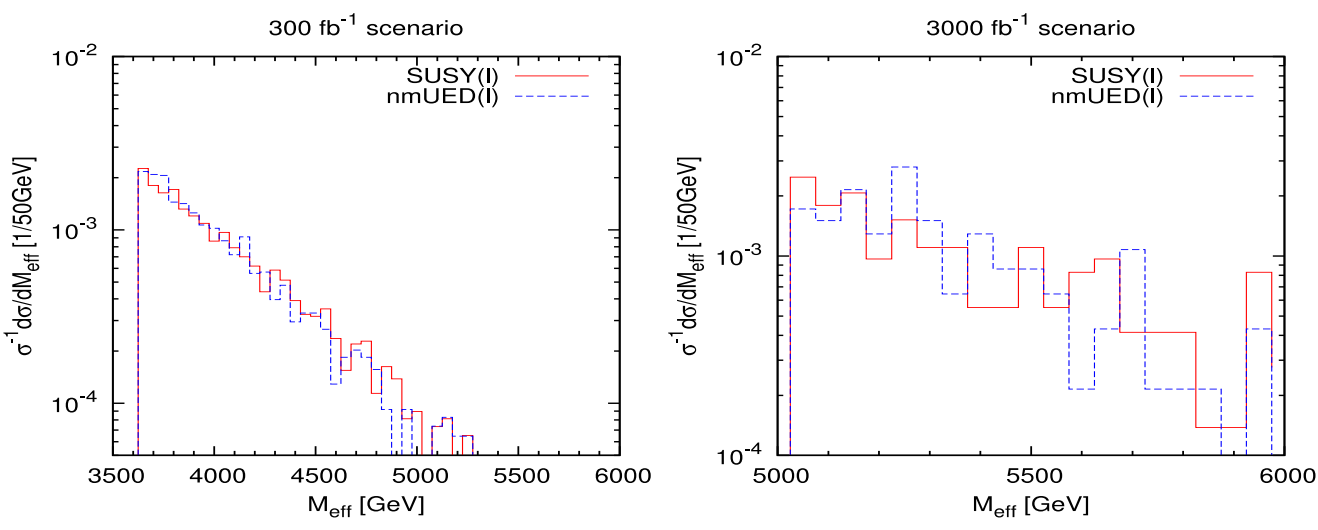

(b)
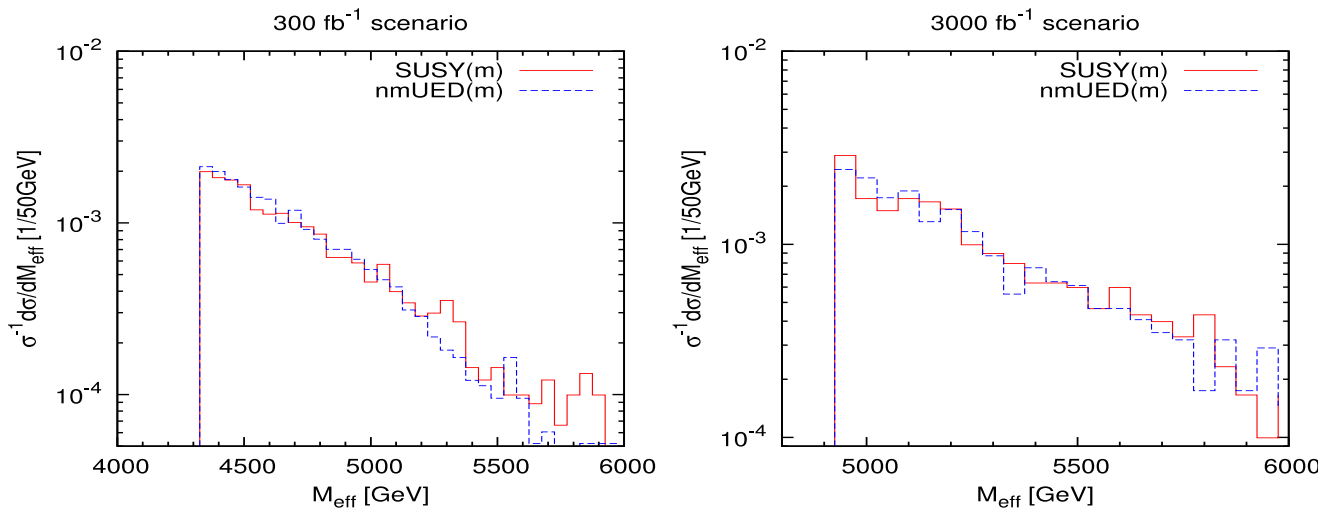

(c)
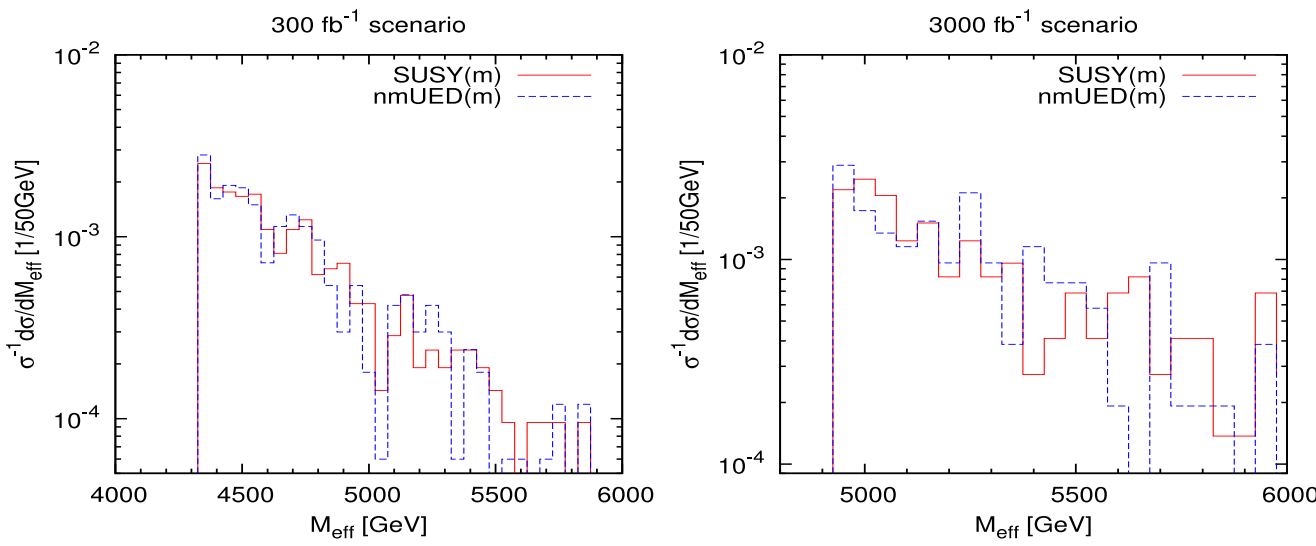

Figure 2 Normalized $m_{\text {eff }}$ distributions of nmUED(blue) and SUSY (red) models. (a) and (b) corresponds to 2J-L signal region generated with BM-1 and BM-3 masses, respectively. (c) and (d) corresponds to 2J-M signal region generated with BM-1 and BM-3 
masses, respectively. For each numeration, the panel on the left and right corresponds to $\mathcal{L}_{\text {int. }}=300$ and $3000 \mathrm{fb}^{-1}$ integrated luminosities, respectively.
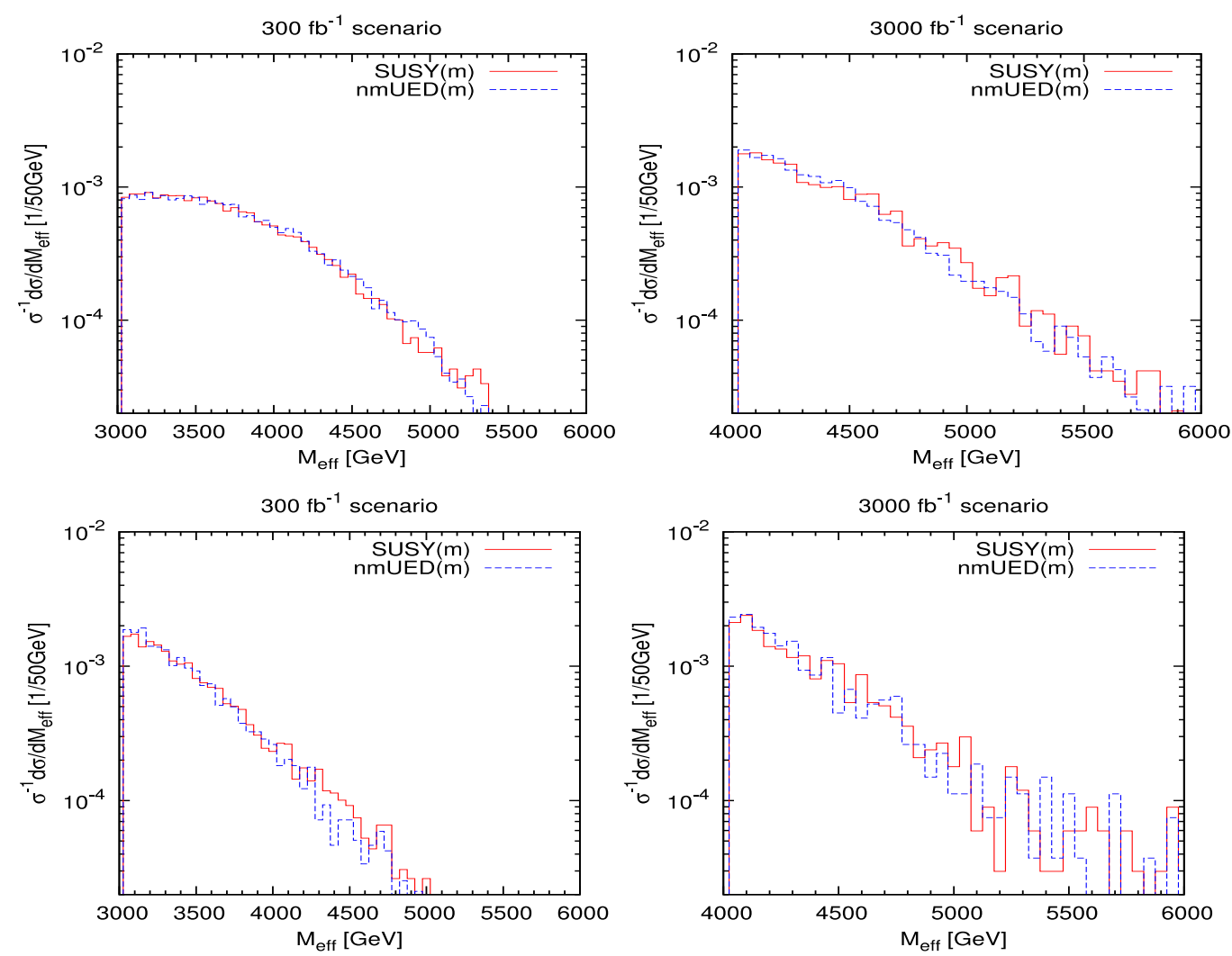

(a)

(b)

Figure 3 Normalized $m_{\text {eff }}$ distributions of nmUED (blue) and SUSY (red) models. (a) and (b) correspond to 3J-M signal region generated with BM-1 and BM-3 masses, respectively. For each enumeration, the panel on the left and right corresponds to $\mathcal{L}_{\text {int }}$. $=300$ and $3000 \mathrm{fb}^{-1}$ integrated luminosity cases, respectively.
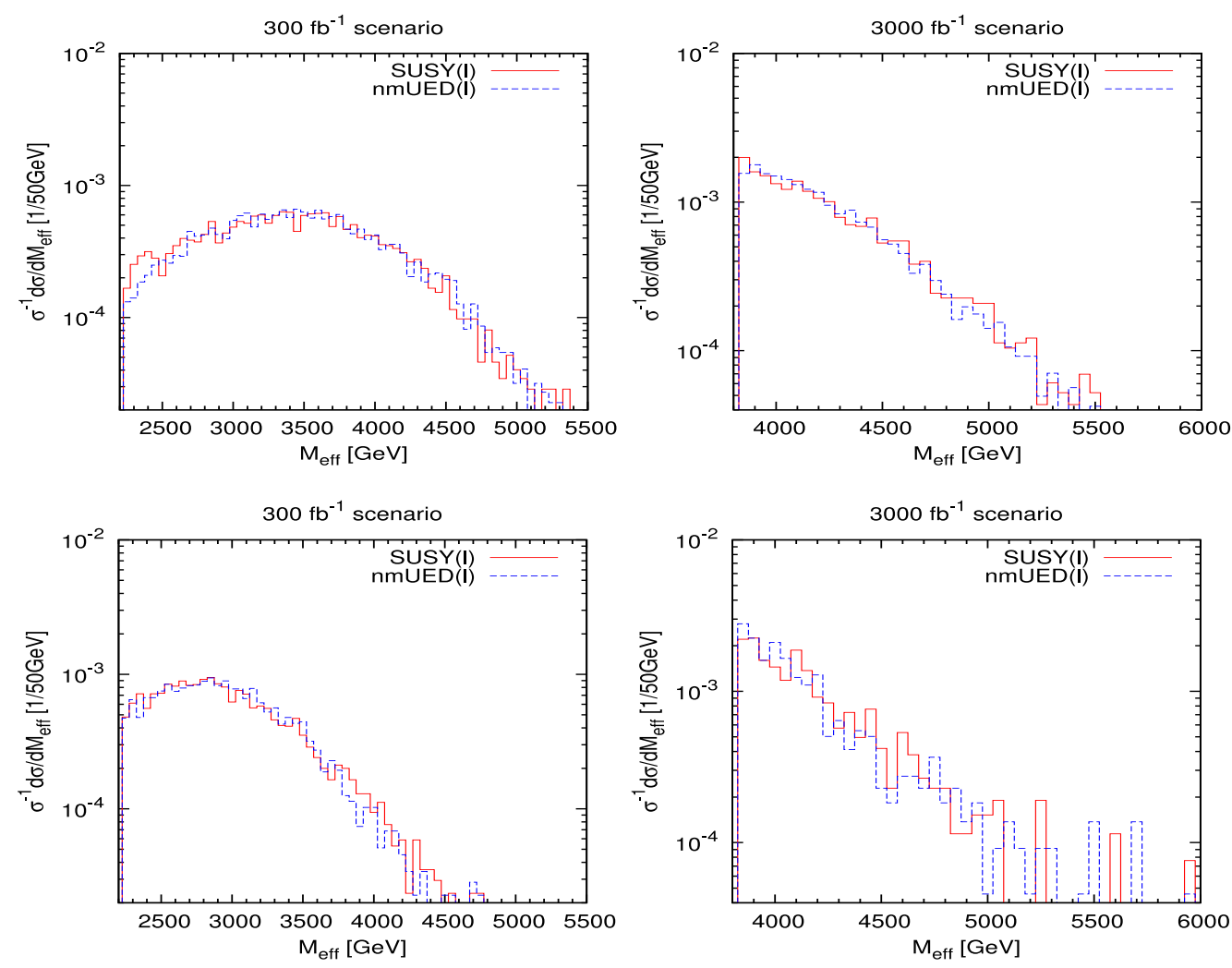

(a) 

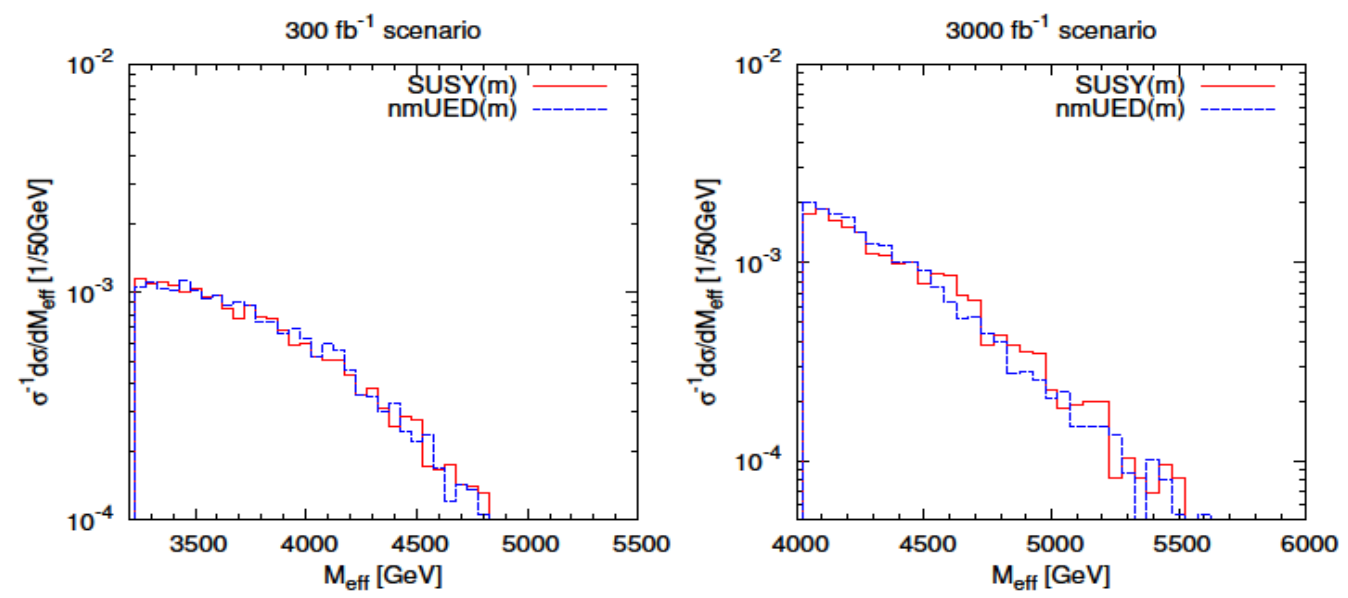

(c)
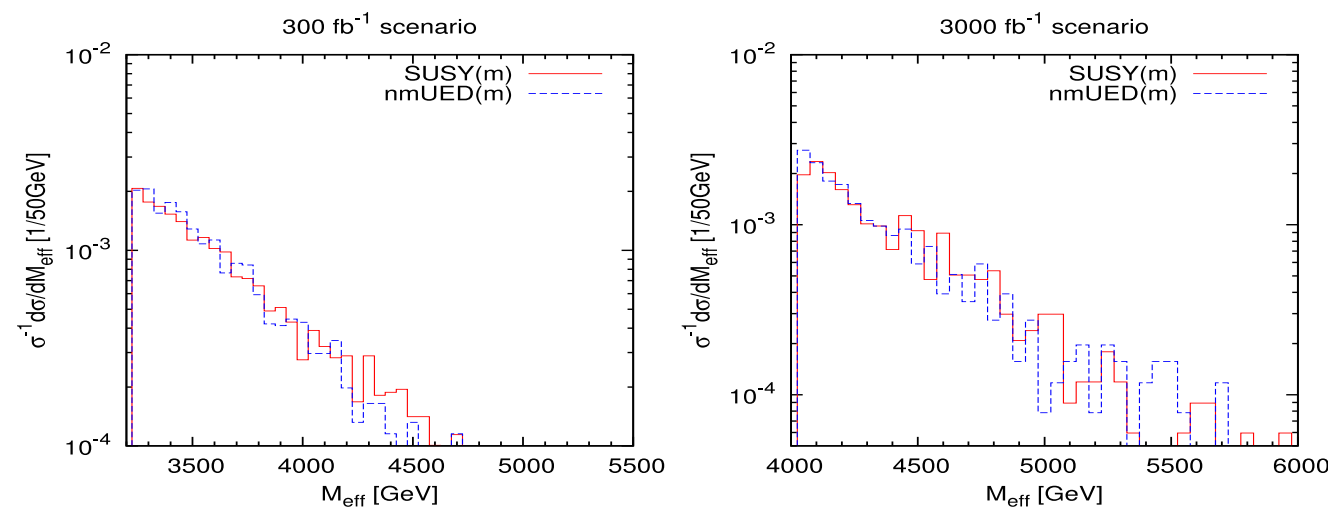

(d)
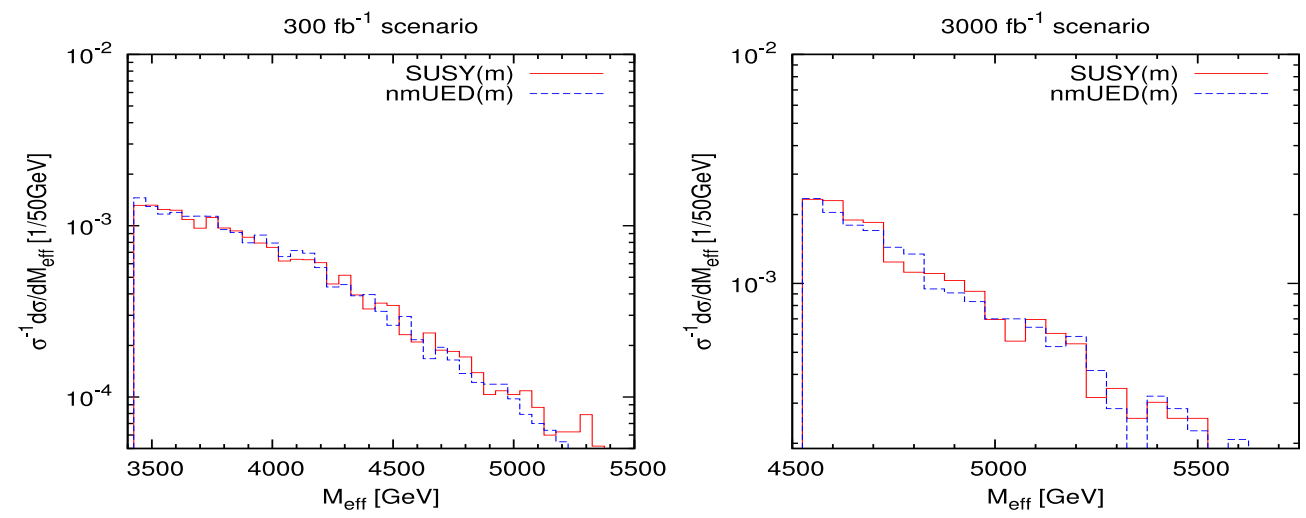

(e)
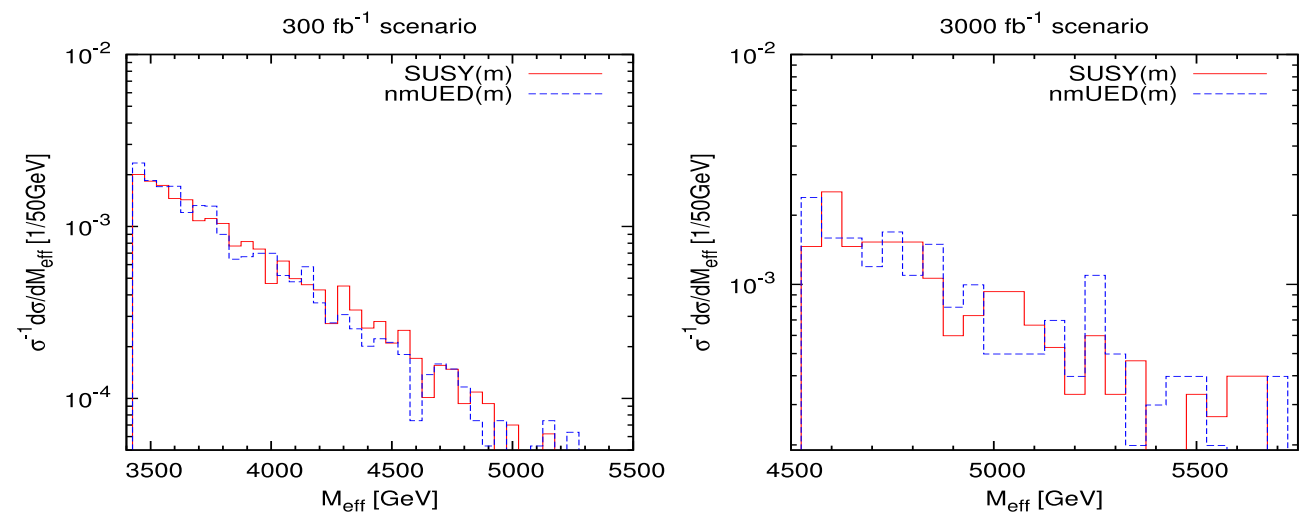

(f)

Figure 4. Normalized $m_{\text {eff }}$ distributions of nmUED (blue) and SUSY (red) models. (a) and (b) correspond to 4J-L signal region generated with BM-1 and BM-3 masses, respectively. (c) and (d) corresponds to 4J-M signal region generated with BM-1 and BM-3 masses, respectively. Similarly, (e) and (f) correspond to 4J-T signal region generated with BM-1 and BM-3 masses, respectively. For each enumeration, the panel on the left corresponds to $\mathcal{L}_{\text {int. }}=300$ and $3000 \mathrm{fb}^{-1}$ integrated luminosities, respectively. 

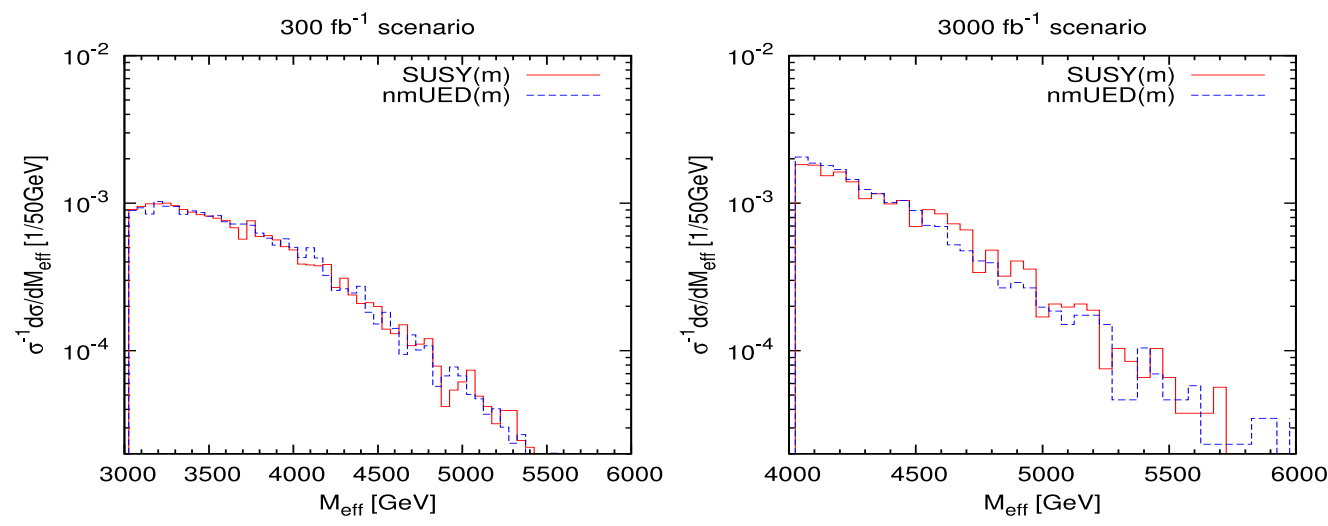

(a)
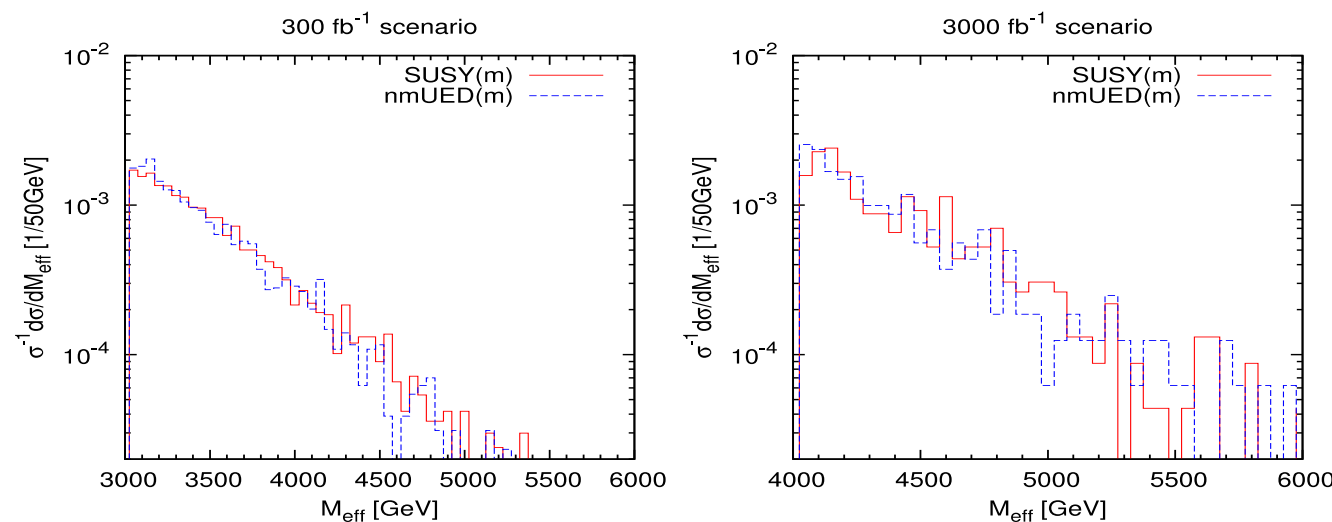

(b)

Figure 5. Normalized $m_{\text {eff }}$ distributions of nmUED (blue) and SUSY (red) models. (a) and (b) correspond to 5J-M signal region generated with BM-1 and BM-3 masses, respectively. For each enumeration, the panel on the left and right corresponds to $\mathcal{L}_{\text {int }}=300$ and $3000 \mathrm{fb}^{-1}$ integrated luminosity cases, respectively.
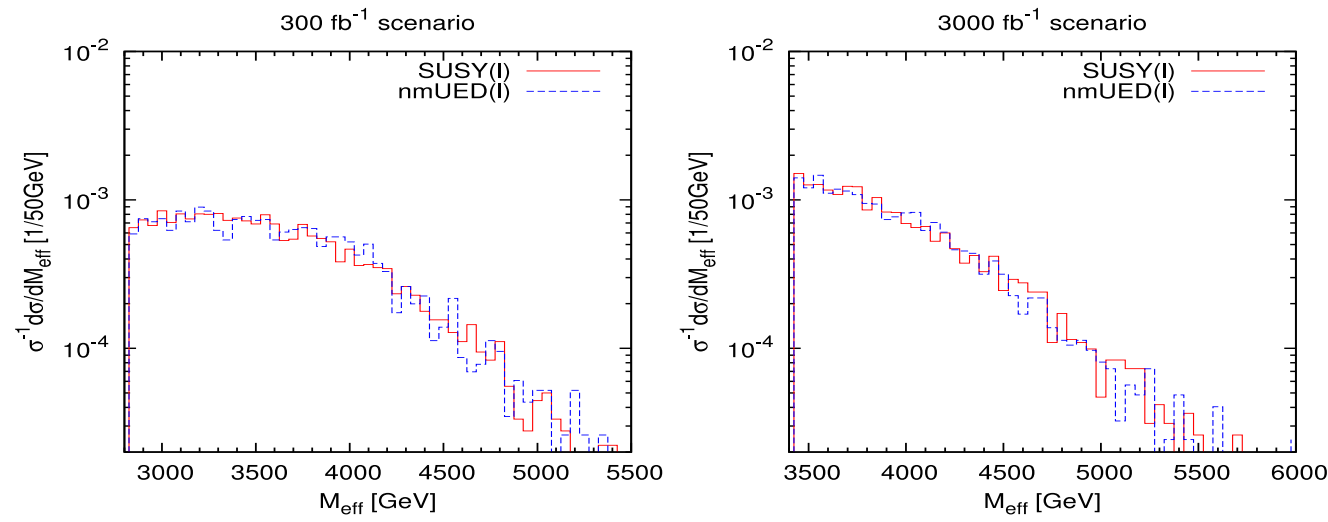

(a)
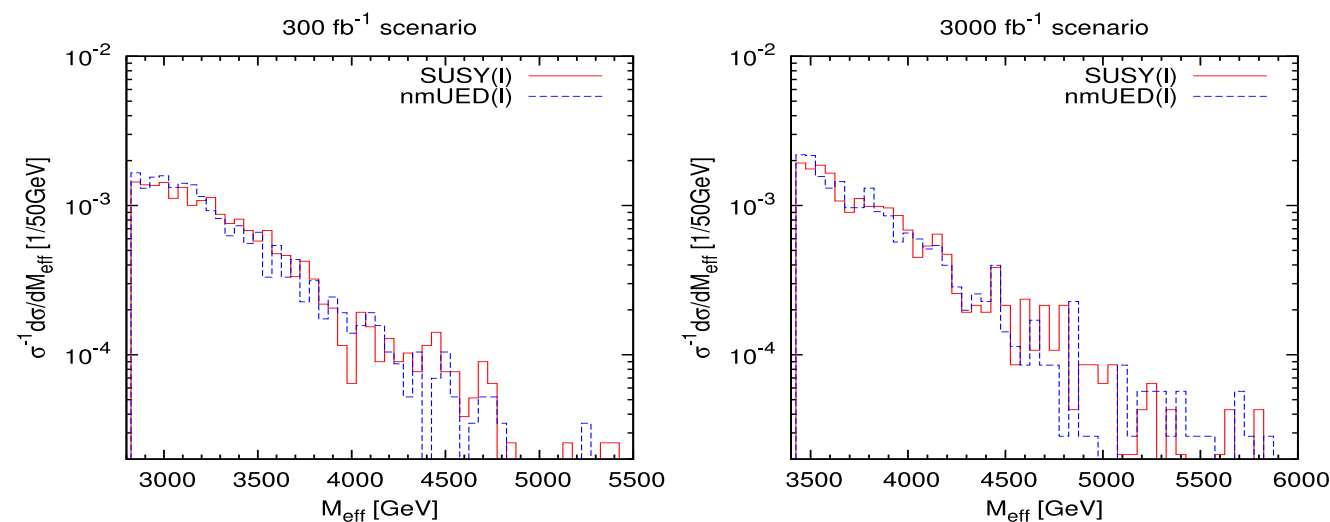

(b) 

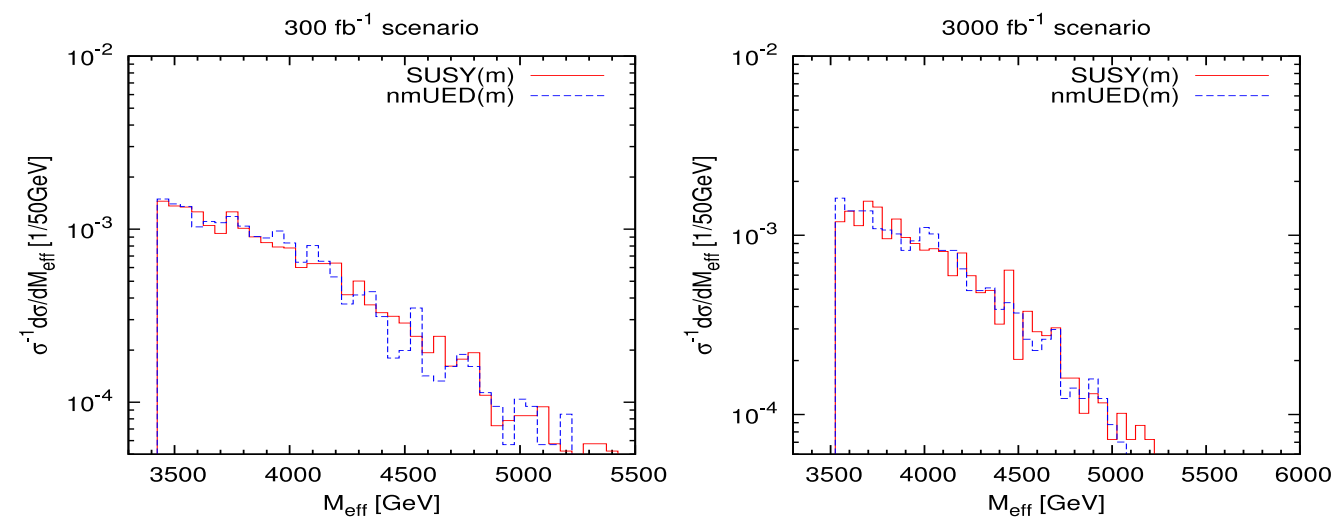

(c)
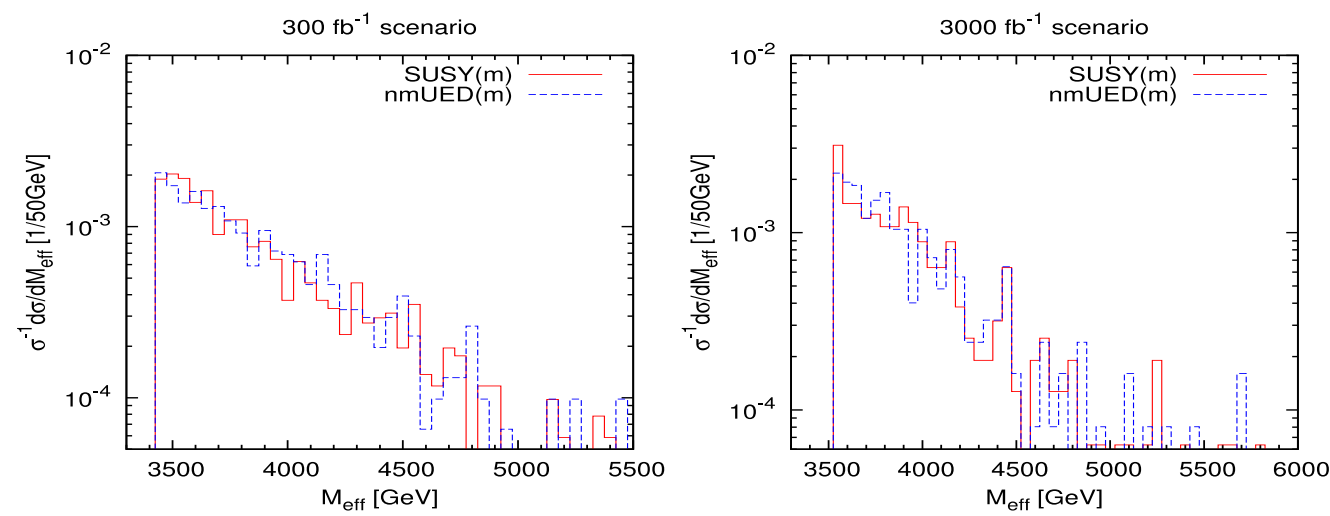

(d)
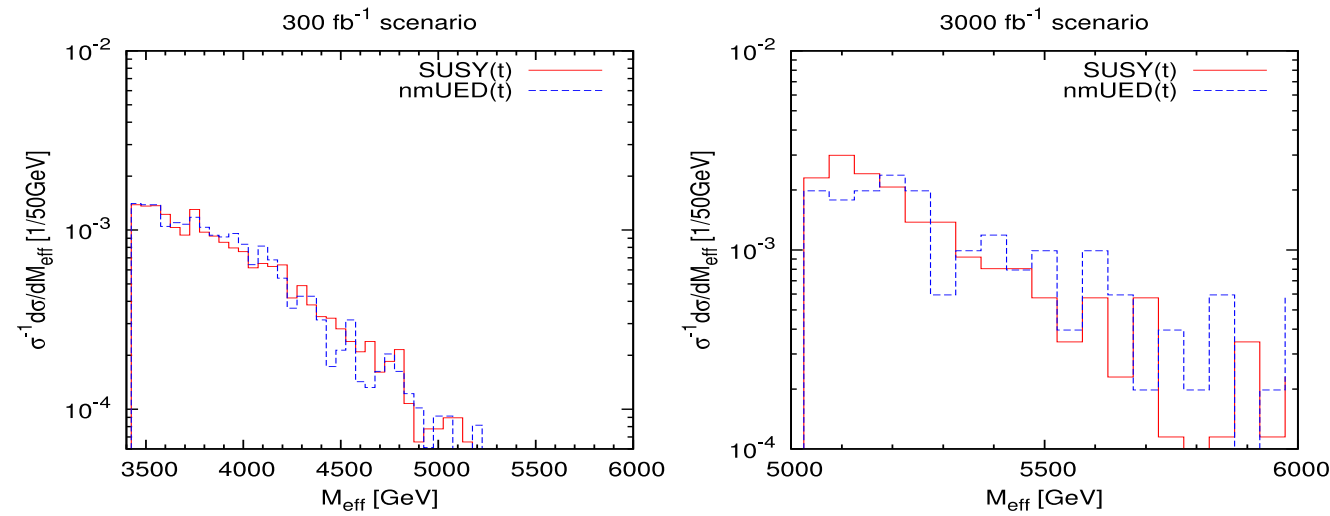

(e)
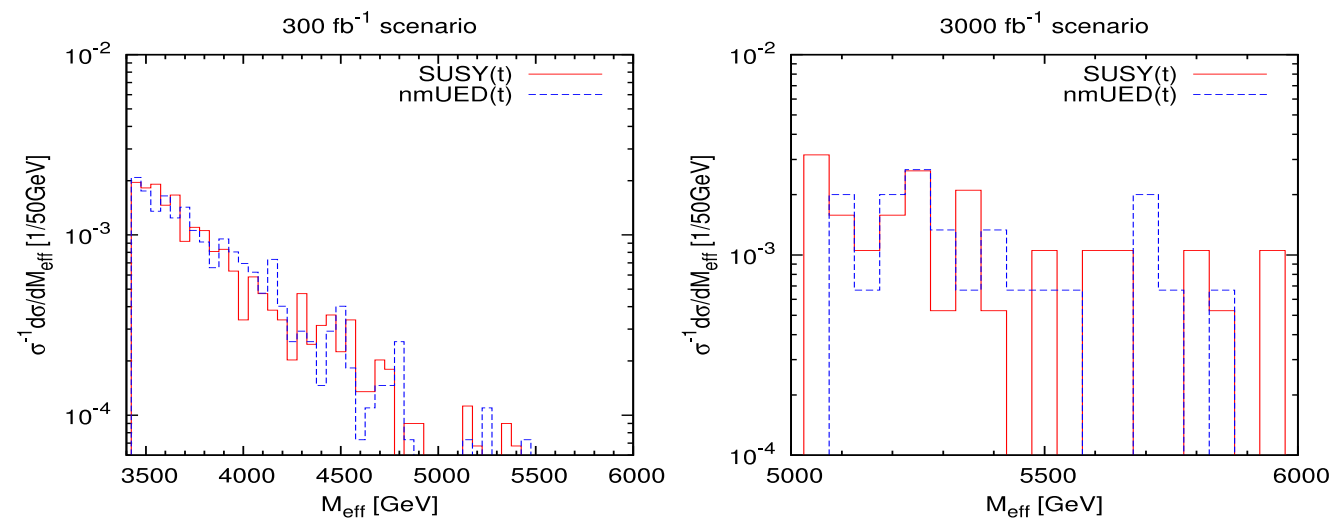

Figure 6 Normalized $m_{\text {eff }}$ distributions of nmUED (blue) and SUSY (red) models. (a) and (b) correspond to 6J-L signal region generated with BM-1 and BM-3 masses, respectively. (c) and (d) corresponds to 6J-M signal region generated with BM-1 and BM-3 masses, respectively. Similarly, (e) and (f) correspond to 6J-T signal region generated with BM-1 and BM-3 masses, respectively. For each enumeration, the panel on the left corresponds to $\mathcal{L}_{\text {int }}=300$ and $3000 \mathrm{fb}^{-1}$ integrated luminosities, respectively. 
Table 1 Cuts used by the ATLAS Collaboration [32] to define the signal regions. $\Delta \phi\left(\right.$ jet, $\left.\vec{E}_{T}^{m i s s}\right)$ is the azimuthal seperations between $\overrightarrow{\mathrm{E}}_{T}^{m i s s}$ and the reconstructed jets. $m_{e f f}$ is defined to be the scalar sum of transverse momenta of the jets together with $E_{T}^{m i s s}$.

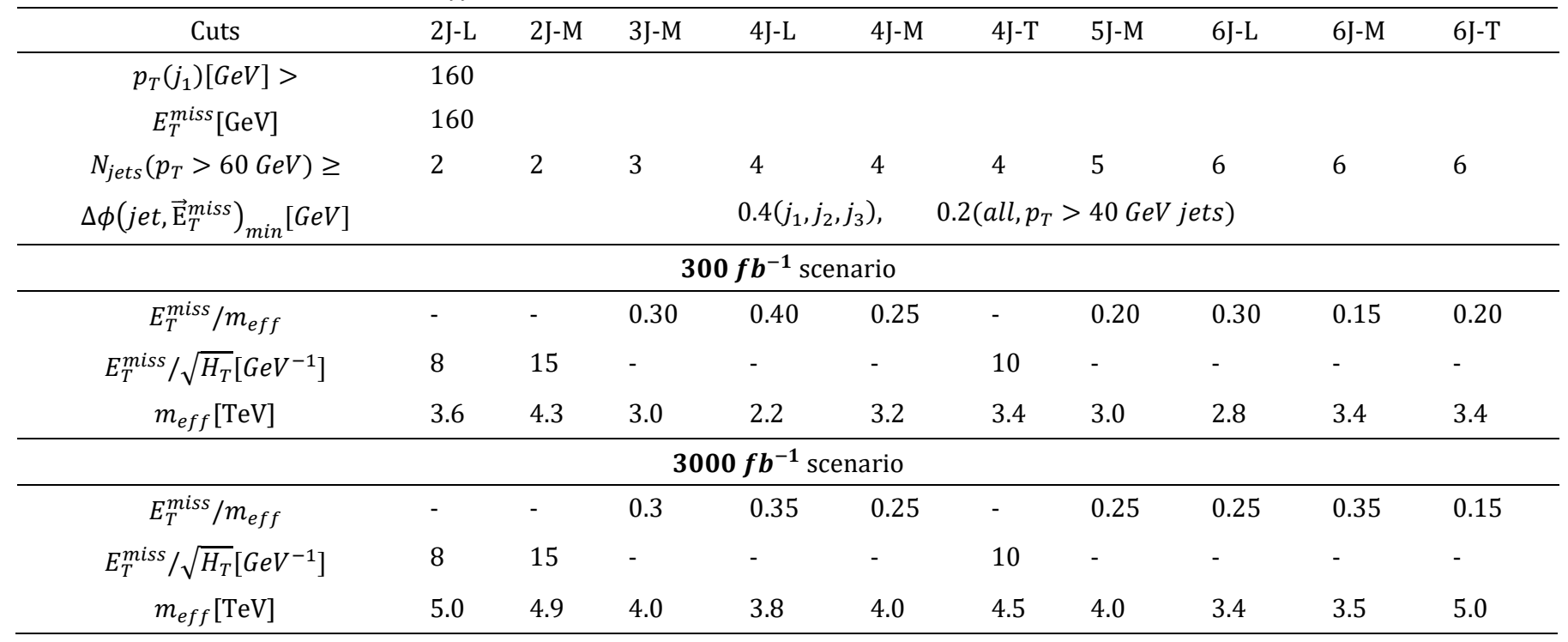

Table 2 Particle mass benchmark points used in comparing SUSY (MSSM) and nmUED signals at $\sqrt{s}=14 \mathrm{TeV}$ with $\mathcal{L}_{\text {int }}=$ $300 \mathrm{fb}^{-1}$ and 3000 integrated luminosities. The first and last column correspond to particles in SUSY and nmUED, respectively, that show similar decay pattern. For nmUED BM-1, 2 and $3, R^{-1}=2,1.8,1.7 \mathrm{TeV}$ is used correspondingly. In all cases, fermion bulk mass term, $\mu$ is set to $-0.02 / L=-0.02 /(\pi R / 2)$. All masses are in $\mathrm{GeV}$ and $r_{x}^{\prime}=r_{x} / L$ where $x=g, \gamma, Z, W$.

\begin{tabular}{|c|c|c|c|c|c|c|c|}
\hline Sparticle & BM-1 & BM-2 & BM-3 & $\begin{array}{l}\text { BM-1 }(\mathrm{nmUED} \\
\mathrm{r} \text { values })\end{array}$ & $\begin{array}{l}\text { BM-2(nmUED } \\
\text { r values) }\end{array}$ & $\begin{array}{l}\text { BM-3 }(n m U E D \\
\text { r values })\end{array}$ & $\begin{array}{ll}\text { Level-1 } & \text { KK } \\
\text { particle } & \\
\end{array}$ \\
\hline$\tilde{g}$ & 2300 & 2000 & 1900 & $r_{g}^{\prime}=-0.210$ & $r_{g}^{\prime}=-0.100$ & $r_{g}^{\prime}=-0.106$ & $g^{(1)}$ \\
\hline$\tilde{\chi}_{1}^{0}$ & 1152 & 1105 & 885 & $r_{\gamma}^{\prime}=0.868$ & $r_{\gamma}^{\prime}=0.719$ & $r_{\gamma}^{\prime}=1.146$ & $\gamma^{(1)}$ \\
\hline$\tilde{\chi}_{2}^{0}$ & 1163 & 1115 & 893 & $r_{Z}^{\prime}=0.850$ & $r_{Z}^{\prime}=0.690$ & $r_{Z}^{\prime}=1.119$ & $Z^{(1)}$ \\
\hline$\tilde{\chi}_{1}^{ \pm}$ & 1163 & 1115 & 893 & $r_{W}^{\prime}=0.850$ & $r_{W}^{\prime}=0.690$ & $r_{W}^{\prime}=1.119$ & $W^{ \pm(1)}$ \\
\hline$\tilde{d}_{L}$ & 2788 & 2244 & 2119 & \multirow{12}{*}{$\begin{array}{l}2800, r_{D}^{\prime}= \\
-0.328 \text { and } \\
r_{U}^{\prime}=-0.328\end{array}$} & \multirow{12}{*}{$\begin{array}{l}2300, r_{D}^{\prime}= \\
-0.230 \text { and } \\
r_{U}^{\prime}=-0.230\end{array}$} & \multirow{12}{*}{$\begin{array}{l}2200, r_{D}^{\prime}= \\
-0.242 \text { and } \\
r_{U}^{\prime}=-0.242\end{array}$} & $d_{D}^{(1)}$ \\
\hline$\tilde{d}_{R}$ & 2841 & 2274 & 2119 & & & & $d_{S}^{(1)}$ \\
\hline$\tilde{u}_{L}$ & 2787 & 2243 & 2118 & & & & $u_{D}^{(1)}$ \\
\hline$\tilde{u}_{R}$ & 2786 & 2213 & 2130 & & & & $u_{S}^{(1)}$ \\
\hline$\tilde{S}_{L}$ & 2788 & 2244 & 2120 & & & & $s_{D}^{(1)}$ \\
\hline$\tilde{S}_{R}$ & 2841 & 2274 & 2201 & & & & $s_{S}^{(1)}$ \\
\hline$\tilde{c}_{L}$ & 2787 & 2243 & 2118 & & & & $c_{D}^{(1)}$ \\
\hline$\tilde{c}_{R}$ & 2786 & 2213 & 2130 & & & & $c_{S}^{(1)}$ \\
\hline$\tilde{b}_{L}$ & 2315 & 2072 & 1985 & & & & $b_{D}^{(1)}$ \\
\hline$\tilde{b}_{R}$ & 2764 & 2426 & 2363 & & & & $b_{S}^{(1)}$ \\
\hline$\tilde{t}_{L}$ & 2374 & 2029 & 1903 & & & & $t_{D}^{(1)}$ \\
\hline$\tilde{t}_{R}$ & 2764 & 2426 & 2363 & & & & $t_{S}^{(1)}$ \\
\hline
\end{tabular}

